\title{
HEMOGLOBINA PLASMÁTICA PREOPERATORIA Y SU CORRELACIÓN CON LA SATURACIÓN REGIONAL DE OXÍGENO DE LA HEMOGLOBINA CEREBRAL
}

Sánchez Valverde José ${ }^{1}$, Hoyos Portal Juan ${ }^{1}$, Fernández Muñoz Carlos ${ }^{1}$, Villalobos Fuenmayor Carlos ${ }^{1}$, Cano Cazeneuve Miguel ${ }^{1}$, Medvedyev, Andriy ${ }^{1}$

1 Anestesiólogo, Clínica Angloamericana.

Introducción: La disfunción cognitiva y la mortalidad post operatoria, están relacionados con bajos niveles transoperatorios de presión arterial, presión de oxígeno arterial, bajos valores de hemoglobina, desaturación regional de oxígeno de la hemoglobina cerebral, entre otros.

Objetivo General: Investigar si existe correlación estadísticamente significativa entre valores de hemoglobina plasmática preoperatoria y la saturación regional de oxígeno de la hemoglobina cerebral. Utilizando sensores NIRS (Espectro de luz cercano al infrarrojo).

Material y Métodos: Se estudiaron 60 pacientes, 39 mujeres y 21 varones, con edades entre 13 y 88 años, ASA I y II, (tabla 1), programados para cirugía electiva, libres de enfermedad cardiovascular, reoperaciones o sangrado preoperatorio. Antes de la inducción anestésica, se colocaron en ambos lados de la frente sensores NIRS SenSmart, Modelo X-100. Registrándose los valores NIRS, los cuales sirvieron como referencia del estudio. Luego de agruparlos de acuerdo a la hemoglobina en forma ascendente, fueron divididos en dos grupos, desde el centro de la muestra, Grupo 1: $\mathrm{Hb}<13,3 \mathrm{~g} / \mathrm{dL}$ y Grupo $2: \mathrm{Hb}>$ a $13,4 \mathrm{~g} / \mathrm{dL}$. Los resultados fueron analizados utilizando la prueba $t$ de Student, con significado estadístico significativo si $\mathrm{p}<$ 0,05 .

Resultados: Los obtenidos Grupo 1: promedio de hemoglobina plasmática de 11,9 , promedio de oximetría regional cerebral 73,2, Desviación estándar (DS) 3,8. (tabla 2) Grupo 2: promedio de hemoglobina plasmática de 15,0 , corresponde un promedio de oximetría regional cerebral de 77,6, DS 3.1. El análisis de la t de Student corresponde una $\mathrm{p}<0,005$.

Conclusiones: Del análisis se obtiene que existe una diferencia estadísticamente significativa entre ambos grupos del estudio, $\mathrm{p}<0,005$. Los valores de $\mathrm{Hb}$ promedio, tienen relación directa con niveles del NIRS. Sería importante considerar alternativas a fin de compensar la disminuida saturación de oxígeno cerebral debido a bajos niveles de hemoglobina. Se propondría ampliar el número de casos.

Tabla 2. Hemoglobina plasmática y su correlación con la saturación regional de $0_{2}$ de la hemoglobina cerebral

\begin{tabular}{|lccccc|}
\hline Promedio & $\mathrm{Sp0}_{2}$ & $\mathrm{Hb}$ & $\mathrm{DS}$ & NIRS & DS \\
\hline Grupo $1: \mathrm{Hb}<13,6$ & 98,7 & 11,9 & 1,4 & 73,2 & 3,8 \\
Grupo 2: $\mathrm{Hb}>13,6$ & 98,7 & 15 & 1,1 & 77,6 & 3,1 \\
\hline
\end{tabular}

